\title{
Bevacizumab treatment for retinopathy of prematurity in South Africa
}

\section{ROP in the developing world}

Retinopathy of prematurity (ROP) is a growing problem in South Africa (SA), as it is in many parts of the developing world. The so-called 'third epidemic' of ROP is caused by a combination of high preterm birth rates, relatively good infant survival and inadequate oxygen monitoring in neonatal facilities. ${ }^{[1]}$ Increasing ROP incidence (due to these factors) has been identified particularly in Latin America and Eastern Europe. SA is generally accepted as having similar challenges in the care of preterm neonates, both in the private and public sectors.

ROP is a fibrovascular complication of poorly vascularised premature retinal tissue. It is mediated by vascular endothelial growth factor (VEGF) and insulin-like growth factor, among others. The main complication of untreated advanced ROP is retinal detachment, leading to blindness in one or both eyes, with potentially major impacts on the health, education and economic potential of the affected individual. ROP occurs exclusively in premature infants, and the SA guidelines recommend screening of all neonates born prior to 32 weeks' gestation and all preterm neonates weighing $<1500$ g. ${ }^{[2]}$

\section{Conventional treatment}

Treatment of advanced ROP has traditionally involved laser photocoagulation of the avascular peripheral retinal tissue, thus reducing the stimulus to retinal neovascularisation as well as fibrosis and scarring. Unfortunately, laser treatment is not without its risks - it is associated with high myopia (often noted many years after the laser treatment), visual field constriction and retinal detachment. The general anaesthesia (GA) typically required for conventional laser treatment carries its own attendant risks in the premature infant. And then, even with optimal laser treatment, visual outcomes are less than ideal. In the 'ETROP'[3] study, application of retinal laser for prethreshold disease showed better outcomes than cryotherapy, but the final visual outcomes were still less than ideal $-25 \%$ of treated eyes having vision worse than $20 / 200$ and $65 \%$ having vision not good enough for a driver's licence. ${ }^{[3]}$

\section{Anti-VEGF treatment}

With this background in mind, it is not surprising that anti-VEGF agents have become so popular in recent years. A single intravitreal injection of anti-VEGF agent, performed under local anaesthesia alone (i.e. without sedation or GA), effectively reverses the ROP neovascularisation process in most cases.

The most commonly used agent, bevacizumab (Avastin, Genentech, USA) is a monoclonal anti-VEGF antibody. It has been shown to be better than conventional laser treatment for posterior (zone I) disease, and equal to laser for so-called 'threshold' disease. ${ }^{[4]}$ The article by Kana et al..$^{[5]}$ demonstrates the effectiveness of bevacizumab in a local SA state hospital setting with an admirable success rate of $95 \%$ in patients with 'threshold disease' (2 failures progressing to retinal detachment out of 43 treated eyes).

The main drivers behind the increasing use of bevacizumab internationally are cost and ease of use. Both are mentioned in the Kana et al..$^{[5]}$ article. In settings where funding is limited, where experienced ophthalmologists are in short supply or where neonatal theatre and high-care facilities are scarce, it makes sense that an option that allows safe, effective, rapid treatment at the bedside is gaining popularity.

\section{Current management of ROP in SA}

Currently, $\sim 50 \%$ of ophthalmologists involved in ROP management in SA report making use of bevacizumab to treat ROP in their practice. A growing number (up to 30\%) make use of anti-VEGF agents as their primary treatment modality (email survey presented at World Society of Paediatric Ophthalmology \& Strabismus Meeting, 2015). The reasons given for use of bevacizumab included 'ease of use' and 'efficacy' as well as 'cost'. Several treating units have no laser device available, and therefore would not be able to treat infants with ROP were it not for the availability of bevacizumab.

Each year, 24000 infants are born in SA at risk of ROP (roughly 1 million live births per year, of whom $2.4 \%$ are preterm $<32$ weeks). Each of these infants should ideally have retinal screening performed 2 or 3 times to rule out ROP. As the number of preterm infants born and surviving in SA continues to rise, the burden of ROP screening and treatment can be expected to follow suit, and the use of antiVEGF agents to treat threshold ROP is likely to gather momentum.

\section{Safety concerns}

Intravitreal anti-VEGF treatment is, however, not without potential problems. The treatment is relatively new and there are still questions about its safety profile. Normal angiogenesis is a key component in the development of the eye as well as other vital systems (lung and brain especially).

Intravitreal anti-VEGF causes regression of the ROP process, but also causes slowing or stalling of the peripheral retinal vascularisation, leaving a permanent avascular zone in some treated infants, with serious concerns that this may lead to later retinal detachment. ${ }^{[6]}$ Also, the slow resolution mandates prolonged weekly surveillance to ensure that there are no late ROP recurrences (up to 60 weeks in some cases).

Underlining fears about systemic safety, bevacizumab has been detected in the serum of both infants and adults after intraocular injection, ${ }^{[7]}$ leading to ongoing debate around appropriate dosage and the best anti-VEGF agent. The dose used by Kana et al. ${ }^{[5]}(0.625 \mathrm{mg})$ is the same as used by most authors in the current literature, although some have recommended a lower dose of $0.25 \mathrm{mg}{ }^{[8,9]} \mathrm{A}$ lower dose would be expected to reduce systemic complications at the expense of a higher risk of recurrence. Conversely, a larger molecule size would be expected to reduce transit out of the eye and therefore also theoretically reduce systemic interactions. This helps to explain why another anti-VEGF agent (ranibizumab; Lucentis, Genentech \& Novartis, USA) with a smaller molecule has not found favour among treating ophthalmologists.

Finally, the risk of endophthalmitis - caused by organisms introduced into the eye at the time of transconjunctival/transscleral injection - is a major concern. This has been shown clearly in the use of anti-VEGF agents to treat macular degeneration, diabetic retinopathy and other forms of macular oedema in adults. Meticulous sterile technique and medication preparation is required to keep this risk as low as possible. ${ }^{[10]}$

\section{Medico-legal implications}

It is unlikely that anti-VEGF agents will become licensed in SA for ROP treatment in the near future (since the number of cases involved 
is insufficient). Therefore, as with any new and developing area, using a so-called 'off-label' medication should be approached with caution. There is a growing body of evidence that anti-VEGF treatment is both effective and safe, but practitioners entering into this arena are advised to carefully document, among other things, available options, informed consent, dosage used, safety precautions employed and follow-up arrangements.

\section{T Pollock}

Division of Ophthalmology, Red Cross War Memorial Children's Hospital and University of Cape Town, South Africa travis.pollock@uct.ac.za

\footnotetext{
1. Gilbert C. Retinopathy of prematurity: A global perspective of the epidemics, population of babies at risk and
implications for control. Hum Dev 2008;84(2):77-82. http://dx.doi.org/10.1016/j.earlhumdev.2007.11.009 implications for control. Hum Dev 2008;84(2):77-82. http://dx.doi.org/10.1016/j.earlhumdev.2007.11.009
Visser L, Singh R, Young M, Lewis H, McKerrow N. Guideline for the prevention, screening and treatment of 2. Visser L, Singh R, Young M, Lewis H, McKerrow N. Guideline for the prevention, screening and treatment of
retinopathy of prematurity (ROP). S Afr Med J 2013;103(2):116-125. http://dx.doi.org/10.7196/SAMJ.6305
}

3. Early Treatment for Retinopathy of Prematurity Cooperative Group, Good WV, Hardy RJ, et al. Final visual acuity results in the early treatment for retinopathy of prematurity study. Arch Ophthalmol 2010;128(6):663671. http://dx.doi.org/10.1001/archophthalmol.2010.34

4. Mintz-Hittner HA, Kennedy KA, Chuang AZ, BEAT-ROP Cooperative Group. Efficacy of intravitreal bevacizumab for stage 3+ retinopathy of prematurity. N Engl J Med 2011;364(7):603-615. http://dx.doi. org/10.1056/NEJMoa1007374

5. Kana H, Mayet I, Soma D, Dawood Alli H, Biddulph S. The efficacy of intravitreal antivascular endothelial growth factor as primary treatment of retinopathy of prematurity: Experience from a tertiary hospital. S Afr Med J 2017;107(3):215-218. http://dx.doi.org/10.7196/SAMJ.2017.v107i3.11080

6. Isaac M, Tehrani N, Mireskandari K. Involution patterns of retinopathy of prematurity after treatment with intravitreal bevacizumab: Implications for follow-up. Eye (Lond) 2016;30(3):333-341. http://dx.doi, org/10.1038/eye.2015.289

7. Wu W-C, Lien R, Liao P-J, et al. Serum levels of vascular endothelial growth factor and related factors after intravitreous bevacizumab injection for retinopathy of prematurity. JAMA Ophthalmol 2015;133(4):391397. http://dx.doi.org/10.1001/jamaophthalmol.2014.5373

8. Avery RL. Extrapolating anti-vascular endothelial growth factor therapy into paediatric ophthalmology: Promise and concern. J AAPOS 2009;13(4):329-331. https://doi.org/10.1016\%2Fj.jaapos.2009.06.003

9. Han J, Kim S, Lee S, Lee C. Low dose versus conventional dose of intravitreal bevacizumab injection for retinopathy of prematurity: a case series with paired-eye comparison. Acta Ophthalmol. Published online: 24 March 2016. http://dx.doi.org/10.1111/aos.13004

10. Mintz-Hittner H. Intravitreal injections of bevacizumab: Timing, technique, and outcomes. J AAPOS 2016;20(6):478-480. http://dx.doi.org/10.1016/j.jaapos.2016.10.002

S Afr Med J 2017;107(3):178-179. DOI:10.7196/SAMJ.2017.v107i3.12388 